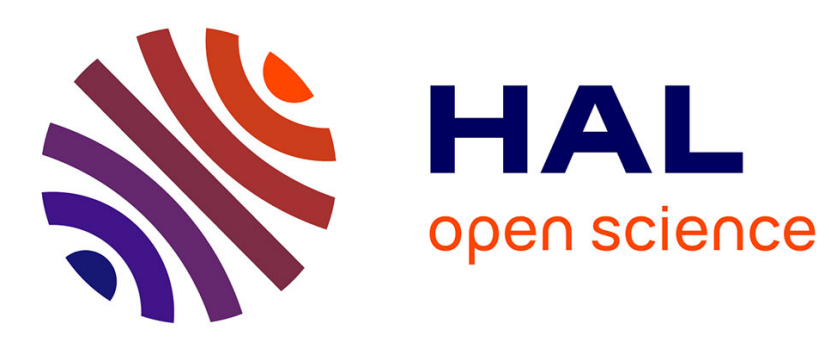

\title{
La philosophie naturelle d'Honoré Fabri (1607-1688)
}

Sophie Roux

\section{To cite this version:}

Sophie Roux. La philosophie naturelle d'Honoré Fabri (1607-1688). Les Jésuites à Lyon, Presses de l'ENS-LSH, pp.25-66, 2005. halshs-00806459

\section{HAL Id: halshs-00806459 \\ https://shs.hal.science/halshs-00806459}

Submitted on 31 Mar 2013

HAL is a multi-disciplinary open access archive for the deposit and dissemination of scientific research documents, whether they are published or not. The documents may come from teaching and research institutions in France or abroad, or from public or private research centers.
L'archive ouverte pluridisciplinaire HAL, est destinée au dépôt et à la diffusion de documents scientifiques de niveau recherche, publiés ou non, émanant des établissements d'enseignement et de recherche français ou étrangers, des laboratoires publics ou privés. 


\title{
LA PHILOSOPHIE NATURELLE D’HONORE FABRI (1607-1688)
}

\author{
Sophie Roux \\ (université de Grenoble II)
}

\section{Introduction 1}

Né en 1607 dans le Bugey, jésuite à 19 ans, Fabri étudia la philosophie (1628-1630) puis la théologie (1632-1636) au collège de la Trinité à Lyon ; il enseigna la grammaire à Roanne (1630-1632), la philosophie à Arles (1636-1640), puis à Lyon (1640-1646)². Il fut alors en correspondance avec Pierre Gassendi, Marin Mersenne, Gilles-Personne de Roberval et Pierre-Jacques Le Tenneur et ses biographes lui attribuent des élèves fameux dans l'histoire des sciences du XVII ${ }^{\mathrm{e}}$ siècle, comme Pierre Mousnier, François de Raynaud, Jean-Dominique Cassini ou Philippe de La Hire ${ }^{3}$. On peut supposer que c'est dans

\footnotetext{
${ }^{1}$ Sur la biographie de Fabri, voir tout d'abord C. Sommervogel, art. "Fabri”, Bibliothèque de la Compagnie de Jésus, 12 vol., Bruxelles/Paris, Oscar Schepens/Alphonse Picard, 1890-1932, vol. III, col. 511-520. J. Brucker, art. "Fabri”, in Dictionnaire de théologie catholique, A. Vacant et E. Mangeot éd., 23 vol., Paris, Letouzey et Ané, 1909-1953, vol. V, col. 2052-2055. H. Beylard, art. "Fabri”, in Dictionnaire de biographie française, R. d’Amat et M. Prévost éd., 19 vol. jusqu’à présent, Paris, Letouzey et Ané, 1929-, vol. XIII, col. 432-434. A. E. Fellman, art. "Fabri”, in Dictionary of Scientific Biography, C. Gillipsie et F. Holmes éd., 18 vol., New-York, Scribners \& Sons, 1970-1990, vol. IV, col. 505-507.
}

${ }^{2}$ Le cursus philosophicus, qui se situe dans les collèges jésuites entre l'enseignement des humanités et celui de la théologie, comprend logique, physique et métaphysique ; c'est dans ces mêmes années que les élèves étudient l'éthique et les mathématiques.

3 Fellman, art. "Fabri”, op. cit., repris partout comme une autorité. C'est évidemment faux pour Cassini $\mathrm{I}^{\mathrm{er}}$ (1628-1712), appelé en France seulement à la fondation de l'Académie des Sciences (1668) ; et on a longtemps ignoré la précocité inouïe de La Hire (1640-1718). Plus sérieusement, outre la Philosophia universa per propositiones digesta rédigée par P. Mousnier, qui sera citée dans cet article, les traces qui nous restent de l'enseignement de Fabri sont des thèses conservées à la Bibliothèque Municipale de Lyon (BML par la suite), que l'on se contentera ici d'énumérer : Bocellini, F., Philosophia per propositiones digesta propugnatas a Francisco Bocellin Bellicensi, Lyon, 1646. Buisson, J.B., Philosophia per propositiones digesta propugnatas a Joan. Baptista Buisson, Lyon, 1646. Mallet, P., Philosophia per propositiones digesta propugnatas a Petro Mallet Lugdunensi, Lyon, 1646. Murard, J. de, Philosophia per propositiones digesta, propugnatas ab Hieronimo de Murard Lugdunensi, Lyon, 1646. Piolle, F., Propositiones mathematicae a Francisco Piolle Manuascensi Lugduni Propugnatae in Coll. Soc. Jes., Lyon, 1645 [également répertoriée sous le titre Theses mathematicae propugnatae a Franc. Piolle, Lugduni in Coll. Soc. Jes.]. Regnauld, F., Philosophia per propositiones digesta propugnatas ab Francisco Regnauld Lugdunensi, Lyon, 1646. Regnauld, F., Theses de universa mathematica propugnatae a Francisco Regnauld, Lyon, 1646. François Regnauld fut donc bien un élève de Fabri, mais le lyonnais appelé François de Regnauld qui exerça les fonctions de Conseiller du Roi (si 
sa période lyonnaise qu'il rencontra Jean Bertet et Claude-François Milliet De Chales, jésuites de la Province de Lyon ${ }^{4}$.

Fin 1646, il fut appelé à Rome ${ }^{5}$ Juste avant cette mutation, un de ses élèves, Pierre Mousnier, avait publié deux tomes de philosophie rédigés d'après ses cours. Les deux événements furent associés à l'époque même, qu'il se soit agi de promouvoir un brillant professeur ou de mettre hors circuit un enseignant trop audacieux ${ }^{6}$. On peut en tout cas établir que les dissensions entre Fabri et ses collègues du collège de la Trinité avaient été cause de troubles à Lyon et qu'il n'enseigna plus après avoir quitté cette ville $^{7}$. En ce sens, son transfert à Rome fut certainement un moyen de le retirer de l'enseignement. Ce ne

c'est bien lui qui a été élève à La Trinité) ne doit pas être confondu, comme le fait Fellman, avec deux auteurs jésuites, Théophile Raynaud et Noël Regnault.

4 Bertet (1622-1692) a enseigné le cartésianisme à Grenoble et, par un de ces paradoxes qui abonderont dans cet article, transmit à Rome l'explication cartésienne de la transsubstantiation qui occasionnera la condamnation des œuvres de Descartes (voir infra note 53) ; il est autrement connu pour avoir été renvoyé de son ordre suite à l'Affaire des Poisons. Pour Milliet de Chasles (1621-1678), de Chambéry, il est l'auteur d'une présentation des Eléments d'Euclide qui fera encore autorité dans l'enseignement des mathématiques au XVIII'

${ }^{5}$ Entre Lyon et Rome, Fabri semble avoir été brièvement prédicateur à Fréjus ; voir Correspondance du P. Marin Mersenne, religieux minime, A. Beaulieu, P. Tannery, C. de Waard éd., 17 vol., Paris, éditions du CNRS, 1932-1988 (par la suite Correspondance de Mersenne), vol. XV, p. 234.

${ }^{6}$ Les deux tomes de 1646 sont la Philosophiae tomus primus qui complectitur : scientarum methodus sex libris explicata, Auctore Petro Mosnerio, cuncta excerpta ex praelectionibus R. P. Honorati Fabri, S. J., Lyon, Jean Champion (par la suite Philosophia) et le Tractatus physicus de Motu locali..., Auctore Petro Mosnerio, cuncta excerpta ex praelectionibus R. P. Honorati Fabri, S. J., Lyon, Jean Champion, 1646 (par la suite De Motu). Avec la Metaphysica demonstrativa sive scientia rationum universalium, Auctore Petro Mosnerio, cuncta excerpta ex praelectionibus R. P. Honorati Fabri, S. J., Lyon, Jean Champion, 1648 (par la suite Metaphysica), ils forment la Philosophia universa per propositiones digesta, et in breve compendium redacta, cum suis momentis rationum. D’après une lettre inédite du père Annat au général des jésuites dit Sommervogel, art. "Fabri”, op . cit., col. 511, les ouvrages de 1646 parurent sans la permission des supérieurs de Fabri. Dans la lettre éditée dans l'appendice II de la Metaphysica, Fabri remercie Mousnier d'avoir transcrit fidèelement son enseignement.

Pour la première interprétation de la mutation de Fabri, voir Mersenne à Torricelli, $1^{\mathrm{er}}$ mars 1647 , in Correspondance de Mersenne, vol. XV, p. 119 : “Honoratus Fabri [...] nuper Romam petiit, ut philosophiam, credo, in Collegio Romano doceat". La seconde trouve son origine dans la lettre de Thibaut citée infra note 7 ; c'est elle que diffusera A. Baillet, La Vie de M. Descartes, 2 vol., Paris, D. Horthemels, 1691, liv. VII, chap. 2, vol. II, p. 300.

7 Thibaut à Mersenne, 3 juin 1647, in Correspondance de Mersenne, vol. XV, p. 245 : “Touchant le Rd Père Fabri votre Révérence saura qu'il est à Rome. A ce que m’assure M. Mousnier, il est traversé par les pères de la Compagnie, et croit-on qu'ils font tout ce qu'ils peuvent pour le faire sortir, comme ils ont fait leur possible pour empêcher l'impression de ses œuvres. Ils firent un jeu de carême prenant, contre les pédants et particulièrement pour se moquer de M. Mousnier qui enseigne à Lyon contre leur gré, mais il y eut des bonnets de Jésuites qui volèrent par là, et tout ce jeu alla en confusion sans pouvoir le parachever, les écoliers et personnes de la ville s’étant soulevés contre eux". 
fut pas pour autant une sanction disciplinaire radicale : il était appelé à Rome pour exercer la fonction délicate et prestigieuse de théologien à la Sacrée Pénitencerie ${ }^{8}$. Il y restera jusqu'à sa retraite, et en sera même promu Recteur, c'est-à-dire directeur.

Cette fonction officielle ne l'empêcha pas de continuer à s'occuper de sciences. On dit qu'il fréquenta des savants italiens (Orazio Grassi, Giovan Battista Baliani, Lorenzo Magalotti, Michel Angelo Ricci) et qu'il fut nommé membre correspondant de l'Accademia del Cimento par le Grand-Duc Léopold II. Il fut en tout cas en relation épistolaire ou polémique avec l'Europe savante (Alfonso Borelli, Robert Boyle, Christian Huygens, Gottfried Wilhelm Leibniz, Henry Oldenburg, Ignace-Gaston Pardies) et publia de nombreux ouvrages sous différents pseudonymes ${ }^{9}$. Une grande partie de ses activités dans sa période romaine fut cependant théologico-politique. Homme de confiance du père François Annat, confesseur de Louis XIV de 1656 à 1670, et ami du cardinal Francesco Albizzi, conseiller du pape Clément IX en matière de jansénisme, il défendit le probabilisme en morale et fut un adversaire acharné des jansénistes ${ }^{10}$. Il prit des positions gallicanes dans l'affaire de la régale, qui lui valurent, on s'en doute,

${ }^{8}$ Sur ce point, voir le témoignage de Le Tenneur, dans une lettre à Mersenne, 9 juillet 1647, in Correspondance de Mersenne, vol. XV, p. 293 : “Mais à propos de Fabri, pourquoi m’écrivez-vous qu'il va être restitué en son honneur et en sa liberté ? A-til donc été dans le crime ou dans l'accusation, il me semble que ce n'a pas été en tout cas une grande punition que d'avoir été fait ensuite Pénitencier de Saint Pierre”. La pénitencerie est un des trois tribunaux de la Curie romaine, spécialisée dans les questions touchant au for interne et échappant à la juridiction des confesseurs ordinaires ; elle atteint son pouvoir maximal au $\mathrm{XVI}^{\mathrm{e}}$ siècle, et, tout au long de son histoire, les papes s'efforcèrent de préciser et limiter ses attributions, qui tendaient toujours à s'étendre. Pour plus de détails sur l'histoire de cette institution, voir Dictionnaire de théologie catholique, op. cit., vol. XII-1, col. 1138-1159.

${ }^{9}$ Il utilise les pseudonymes d'Antimus Conygius pour sa défense du quinquinna (Pulvis Peruvianus vindicatus de ventilatore ejusdem suscepta defensio ab Antimo Conygio hortatu Germani Poleconii, Rome, François Corbellet, 1655) et d'Antimus Farbius dans un ouvrage sur les sinus (Opusculum Geometricum de linea sinuum et cycloide, Rome, François Corbellet, 1659). Pour attaquer la manière dont Huygens interprétait ses propres observations de l'anneau de Saturne, il se sert du nom d'Eustache Le Divin, le fabricant de lunettes qui le fournissait à Rome (Eustachii De Divinis Septempedani Brevis Annotatio in Systema Saturninum Christiani Eugeni, Rome, Jacob Dragonelli, 1660, Eustachius De Divinis Septempedanus pro sua Annotatione in Systema Saturninum Christiani Eugenii adversus ejusdem assertionem, Rome, Jacob Dragonelli, 1661, passim. On peut suivre le développement de cette controverse in Euvres complètes de Christian Huygens, Société hollandaise des sciences éd., 22 vol., La Haye, 1888-1950 (par la suite Euvres de Huygens), vol. III et vol. IV). Qu'il ait adressé une lettre d'hommage à Louis XIV sous le pseudonyme d'Honoré Fabart di Zizan (Hercole vero, Lyon, c. 1660) montre que cet usage n'était pas réservé aux ouvrages scientifiques et qu'il n'était pas motivé par la crainte de la censure.

10 Sur ce point, voir Mémoires du Père Rapin, 3 vol., Paris, 1865, vol. III, p. 237, cité in F. Azouvi, Descartes et la France. Histoire d'une passion nationale, Paris, Fayard, 2002, p. 25-26. L'œuvre théologique de Fabri ne sera pas analysée ici ; signalons tout de même que la BML conserve plusieurs manuscrits qu'il serait intéressant de publier, par exemple et pour ne citer que les textes en français, "La Synopse du Jansénisme”, in Oeuvres du Père Honoré Fabri, BML, mss $701-704$ (par la suite Oeuvres de Fabri), vol. II, f. 206 sqq., "De la Question De auxiliis", in Oeuvres de Fabri, vol. IV, f. 55 sqq., 
certaines inimitiés romaines ${ }^{11}$. En 1680, il fut relevé de ses fonctions et se retira dans les environs de Rome. Il y mourut en 1688.

C'est dès lors à trois titres au moins qu'il était normal, dans un colloque consacré aux jésuites à Lyon, qu'un historien des sciences évoque Fabri : il fut jésuite, il fut lyonnais, il fut savant. La motivation de l'article qu'on va lire est cependant ailleurs : il s'agit de considérer son œuvre comme un témoin des mutations de la philosophie naturelle au XVII ${ }^{\mathrm{e}}$ siècle. On entend par "philosophie naturelle" durant toute cette période l'étude de la nature ; par delà la permanence d'une telle définition, cette discipline connaît cependant une mutation. Au début du XVII ${ }^{\mathrm{e}}$ siècle, faire de la philosophie naturelle, c'est principalement commenter des textes aristotéliciens, qu'il s'agisse de physique générale (décrire les principes de la nature au sens aristotélicien comme la matière et la forme, les quatre causes, le changement, etc.), de physique particulière (se demander, par exemple, pourquoi un corps rebondit, ou encore, d'où viennent les pouvoirs de l'aimant) ou de questions qui, pour nous, sont de l'ordre de la physiologie, de la psychologie ou de la philosophie de l'esprit. A la fin du XVII ${ }^{\mathrm{e}}$ siècle, la philosophie naturelle peut éventuellemment comprendre des discussions sur les principes, mais ce ne sont plus les mêmes (matière et mouvement constituent des alternatives plausibles aux principes aristotéliciens, voire les ont remplacés) ; la part belle est faite à des expériences plus ou moins récentes et plus ou moins extraordinaires ; enfin, les mathématiques ont leur rôle à jouer, sous diverses formes d'ailleurs.

L'historiographie de ces vingt dernières années n'a pas remis en cause l'existence de cette mutation, mais plutôt sa description comme une Révolution Scientifique. Ce qui se disait autrefois en effet, c'est qu'il y avait eu "Révolution Scientifique" : une révolution de la science toute entière et non simplement l'évolution de telle ou telle pratique savante ; une révolution supposant une transformation des fondements métaphysiques des sciences. On disait en particulier que les principes clairs des "nouveaux philosophes" s'étaient substitués aux entités barbares d'obscurs professeurs "scolastiques". Or les contours de cette opposition ont commencé à se brouiller ces vingt dernières années. Le lien entre les principes de la philosophie naturelle et les deux sciences supposées être au cœur de cette révolution, la mécanique et l'astronomie, s'est relâché, en particulier parce qu'on a plus travaillé sur la pratique aristotélicienne des sciences mixtes ${ }^{12}$. L'intérêt pour les minores a montré que les catégories de

“Observations sur un certain traité dans lequel on réfute Jansénius", in ibid., f. 66 sqq., "De la Prédestination”, in ibid., f. 86 sqq., "De l'Eucharistie”, in ibid., f. 100 sqq., "De la Grâce”, in ibid., f. 110 sqq., "De l'Oraison de quiétude”, in ibid., f. 136 sqq.

11 Voir sur ce point le témoignage de Fabri lui-même in Oeuvres de Fabri, vol. I, f. 236, f. 257-258.

12 Les études décisives sur ce point ont sans doute été celles d’Ugo Baldini sur Clavius et le Collegio Romano ; voir en particulier Legem impone subactis. Studi sulla filosofia e scienza dei Gesuiti in Italia, 1540-1632, Rome, Bulzoni, 1992, et Christoph Clavius et l'attività scientifica dei Gesuiti nell'età di Galileo. Atti del Convegno internazionale (Chieti, 28-30 aprile 1993), Rome, Bulzoni, 1995. 
"philosophie nouvelle" et de "philosophie scolastique" n'étaient ni distinctement séparées l'une de l'autre, ni suffisantes pour couvrir le champ des positions possibles en philosophie naturelle ${ }^{13}$.

L'œuvre de Fabri est intéressante précisément parce qu'elle remet en question les catégories historiographiques qui continuent d'avoir cours sans qu'on y pense ; elle constitue un bon témoin dans le contexte d'une réflexion sur la question suivante :

L'aristotélisme était une tradition souple, qui s'était pendant des siècles adaptée à de nouveaux problèmes : quelles sont les perturbations induites par les sciences et la philosophie nouvelles au XVII siècle qu'il ne toléra pas?

Ou, pour formuler la chose par son autre bout :

Les cuvres des novateurs furent lues par des scolastiques qui n'étaient pas des obscurantistes enfermés dans un camp retranché : une telle réception a-t-elle infléchi le sens que la postérité a conféré à ces ouvres?

Fabri constitue un bon témoin parce qu'il s'est effectivement situé aux frontières, soutenant des positions qui nous paraissent aujourd'hui contradictoires : qu'elles aient pu à un moment ne pas être perçues comme telles, c'est ce que montrera dans un premier temps une analyse de sa renommée. Certaines thèses décisives de Fabri dans les champs polémiques suscités par la science nouvelle et la philosophie nouvelle, respectivement incarnées par Galilée et par Descartes, seront ensuite examinées. Pour finir, il restera à préciser son rapport à Aristote et aux scolastiques.

\section{Fabri au miroir de la renommée}

Ce sont des lettres adressées à Gassendi et Mersenne qui, en 1643, firent entrer Fabri dans le monde savant. Défendant le géocentrisme et soutenant que les espaces traversés dans des intervalles de temps

\footnotetext{
${ }^{13}$ Le travail de C. B. Schmitt a été séminal dans la réévaluation de l'aristotélisme ; voir, par exemple, Aristotelianism and the Renaissance, Cambridge, Mass., Harvard U.P., 1983. Plus récemment, voir T. Sorell éd., The Rise of Modern Philosophy, Oxford, Oxford U.P., 1993. C. W. T. Blackwell et S. Kusukawa éd., Philosophy in the Sixteenth and Seventeenth Centuries. Conversations with Aristotle, Aldershot/Brookfield/Singapore/Sydney, Ashgate, 1999. R. Ariew, Descartes and the Last Scolastics, Ithaca/London, Cornell U.P., 1999. C. Leijenshorst, C. Lüthy et J. M. M. H. Thijssen éd., The Dynamics of Aristotelian Natural Philosophy from Antiquity to the Seventeenth Century, Leide, Brill, 2002. Pour des présentations synthétiques de la "physique jésuite", voir L. W. B. Brockliss, "Pierre Gautruche et l'enseignement de la philosophie de la nature dans les collèges jésuites français vers 1650”, in L. Giard éd., Les Jésuites à la Renaissance. Système éducatif et production des savoirs, Paris, P.U.F. 1995, p. 187-220. U. Baldini, “The development of Jesuit 'physics' in Italy, 1550-1700: a structural approach", in Blackwell et Kusukawa éd., Philosophy in the Sixteenth and Seventeenth Centuries, op. cit, p. 248279, en particulier les p. 269-270 consacrées à la Physica.
} 
successifs et égaux par des corps en chute libre sont comme la suite des nombres naturels (et non comme la suite des nombres impairs), Fabri est assurément un adversaire de Galilée, que Gassendi comme Mersenne avaient alors entrepris de défendre. Mais c'est un de ces adversaires estimables qui contribuent à promouvoir le paradigme supposé par la science galiléenne ${ }^{14}$. Fabri revendique en effet deux thèses essentielles à ce paradigme : thèse méthodologique selon laquelle "la communion des choses physiques et mathématiques est telle qu'il n'y a presque aucun traité en physique qui puisse se passer de mathématique" ; thèse ontologique selon laquelle "dans toute sensation, quelque mouvement local se produit" 15 .

C'est précisément parce que Gassendi et Mersenne ne perçurent pas le travail de Fabri comme une remise en cause du paradigme galiléen dans sa généralité qu'ils l'encouragèrent. Gassendi voulait lui faire publier ses "essais admirables" et ses "méditations si nombreuses et tellement plus lumineuses que les enseignements de la philosophie commune" ; ayant échoué dans cette entreprise, il se tourna vers Mousnier pour la mener à bien ${ }^{16}$. Mersenne se chargea d'obtenir le privilège et, dans sa correspondance, il ne tarit pas d'éloges sur Fabri et sa philosophie ${ }^{17}$. Les publications des années 1640 correspondent donc

\footnotetext{
${ }^{14}$ La notion de "paradigme galiléen" n'est pas équivalente ici à la thèse historique que Galilée aurait mis en place quelque chose d'inouï avant lui (voir les références données supra note 12). Il s'agit seulement de constater qu'il a exemplairement illustré une manière de traiter les choses physiques, qui pouvait, à un certain degré de généralité, être appréciée et exploitée même par ses contradicteurs.
}

15 Fabri à Gassendi, 9 août 1643, in Correspondance de Mersenne, vol. XII, p. 276-277 : "Quamvis enim pura illa mathesis valde placeat, nuda tamen et exsucca est, nec ita me afficit ut alia quae cum re physica conjuncta non animum modo, sed etiam sensus ipsos delectat; et vero tanta est rerum physicarum et mathematicarum communio, ut nullus fere sit in Physica tractatus qui mathesi carere possit [...]. Illud magis notum est quod sensu [...] percipimus quam quod abstractive, id est analogice intellegimus, itaque ab iis accidentibus initium ducendum esse existimo quae sensibus percipimus; et cum in omni sensu fit aliquis motus localis, de motu locali primo agendum esse puto". Sur l'union des mathématiques et de la physique, voir aussi Mousnier à Mersenne, $1^{\mathrm{er}}$ mai 1646, in ibid, vol. XIV, p. 264, et, dans le Comparationum tractatus, la "Comparatio prima Physicam inter et Gemometriam", in Oeuvres de Fabri, vol. IV, f. 203-245.

16 Gassendi à Mousnier, $1^{\mathrm{er}}$ octobre 1645, début de la Philosophia, n.p. : "Tertius scilicet praetercurrit annus, ex quo cum mihi concessum fuisset egregia quaedam specimina scriptorum ejus conspicere, interpellare virum coepi, ac non modo per literas, sed etiam per viros vere nobiles germanum ejus ac patruelem Valgelasium sollicitare, ut quae tam multa, tamque praeclara supra vulgare philosophandi institutum excogitasset, publici juri faceret ; enimvero ea semper fuit animi ipsius modestia, ut et hactenus cecinerim surdo, et pene ut in posterum id ab eo obtineam, desperem".

17 Pour l'obtention du privilège, voir Fabri à Mersenne, 2 avril 1646, et Mousnier à Mersenne, 1 er mai 1646, in Correspondance de Mersenne, vol. XIV, resp. p. 192 et p. 264. Pour les éloges, voir Mersenne à Descartes, [22] mars 1646, in ibid., vol. XIV, p. 166 : "Quant au P. Fabri de Lyon, qui enseigne la philosophie et mathématique en honnête homme et qui commence à faire imprimer ses 18 volumes [sic] pour contenir tout le corps philosophique et mathématique, il a un excellent esprit et certainement il est pour nous donner de belles choses. [...] Enfin c'est un géant en science”. Mersenne à Mousnier, 5 novembre 1645, début de la Philosophia, n.p. : "Vix explicare possum quanta fuerim affectus animi voluptate, statim atque 
à un moment où les savants "novateurs" considèrent que Fabri est à leurs côtés. C'est conséquemment une réputation de bon philosophe et de bon mathématicien qui accompagna Fabri lorsqu'il partit pour Rome ${ }^{18}$.

La deuxième moitié des années 1660 constitue un pic dans les publications de Fabri. Son intention semble avoir été de relancer le processus éditorial que son départ pour Rome, mais sans doute aussi les réticences de son libraire devant l'ampleur de l'effort demandé, avaient interrompu ${ }^{19}$. Comme il s'en explique lui-même, deux traités sur les êtres vivants lui permirent de sonder le gué ${ }^{20}$. Le bon accueil qui leur fut réservé le convainquit de reprendre les traités de physique, annoncés mais non publiés une bonne dizaine d'années plus tôt ${ }^{21}$. En ce sens, les ouvrages des années 1660 réalisent le projet de la fin des années 1640. Entretemps cependant, les mutations de l'espace philosophique et scientifique avaient été considérables en France ; pour le dire en un mot, le gros des troupes n'est plus sur le front galiléen, mais sur le front cartésien : c'est le moment où fait rage la guerre à l'issue de laquelle l'œuvre de Descartes deviendra en France la référence en matière de nouveauté, l'index en fonction duquel les œuvres des uns et des autres seront classées sur l'axe qui mène de l'ancien au nouveau'22.

didici te philosophiam illam subtilissimam et solidissimam prelo tandem commitere, quae vel ipsos Pyrrhonios fateri cogat e Democriti puteo nunc veritatem emergere".

18 Baliani à Mersenne, 31 mars 1647, in Correspondance de Mersenne, vol. XV, p. 147 : "E stato qui il P. Onorato Fabro con cui ho trattato con multo gusto, e mi pare un huomo molto dotto, e vedo che in molte cose habbiamo assai dato nel'istessi pensieri”. Ricci à Torricelli, [1er juillet 1647], in ibid., p. 285 : “Qui abbiamo un Padre Gesuita franceze, detto il Padre Fabri, stimato per matematico valoroso, secondo mi dicono gli amici miei che lo conoscono per fama".

19 Thibaut à Mersenne, 15 octobre 1647, in Correspondance de Mersenne, vol. XV, p. 477 : “Son libraire m'a dit qu'il n'avait pas la volonté d'imprimer les autres tomes de la philosophie et qu'il ne pouvait débiter, y ayant 14 tomes".

20 Tractatus duo, quorum prior est de Plantis et de Generatione Animalium, posterior De Homine, Paris, François Muguet, 1666 (par la suite Tractatus duo). Dans la préface, n.p., Fabri s'explique sur la différence entre l'ordre logique et l'ordre qu'il a suivi dans son édition.

21 Dans la préface de la Philosophia, n.p., Mousnier annonce la publication de quatorze tomes : trois des quatre premiers correspondent aux ouvrages de 1646-1648 ; les sept suivants aux Tractatus duo et à la Physica, id est De scientia rerum corporearum in decem tractatus distributa, 5 vol., Lyon, Laurent Anisson, 1669-1671 (par la suite Physica) ; je n'ai pas cherché à établir la correspondance entre les deux derniers, consacrés à la théologie naturelle et à la morale, et des ouvrages publiés par Fabri.

22 Dans les années 1660-1680, alors même que les pouvoirs politiques et religieux condamnent l'enseignement des thèses cartésiennes, le cartésianisme devient une doctrine à la mode dans les salons et chez les Grands, les œuvres de Descartes d'abord publiées en Hollande sont rééditées à Paris, celles qu'il n'avait pas publiées de son vivant sont éditées (la mécanique et la musique par Nicolas Poisson, la correspondance par Clerselier, l'Homme et Le Monde par Louis de La Forge), dans tous les domaines enfin paraissent des traités gagnés à la cause cartésienne. Voir sur ce point F. Bouillier, Histoire de la 
Les œuvres ambivalentes de Fabri donnent dès lors lieu à deux espèces de réception. La première est positive, Fabri serait un "novantique" : un philosophe qui a su prendre le meilleur des anciens et des nouveaux ${ }^{23}$. C'est par exemple le cas dans le compte rendu que le Journal des Savants donna en 1666 de ses traités sur les êtres vivants :

\begin{abstract}
De ceux qui ont excellé dans la connaissance des choses naturelles, les uns se sont attachés à Aristote avec tant de scrupule qu'ils n'ont rien voulu recevoir que ce qu'il a enseigné, les autres s'en sont éloignés avec tant de passion qu'ils ont entièrement rejeté sa doctrine ; et comme les premiers ont quelquefois mieux aimé être du parti d'Aristote que de celui de la vérité, les derniers ont souvent mieux aimé n'être pas du parti de la vérité que d'être de celui d'Aristote. Le P. Fabri a tenu le milieu entre ces deux extrémités ${ }^{24}$.
\end{abstract}

Cette réception n'est pas propre à la France, mais se retrouve, mutatis mutandis, dans d'autres contextes nationaux. C'est comme un "éclectique" que Fabri apparaît dans la Philosophia eclectica de Joseph Christoph Sturm (1679) ; dans son Polyhistor historicus (1688-1692), Daniel Georg Morhof le classe avec Jean De Raey et Adrien Heereboord parmi les "novateurs" qui ont réussi à concilier Aristote et Descartes 25 .

Mais pour certains, et leur nombre ira croissant à partir des années 1680, la conciliation des anciens et des nouveaux était à peu près aussi chimérique que la réunion des églises : entre scolastiques et cartésiens, il fallait choisir son camp. Bien plus, écrire de longs et pesants ouvrages en latin, comme le faisait Fabri, c'était appartenir à un autre âge, le nouveau public exigeant des opuscules en français, aussi

philosophie cartésienne, 2 vol., 3ème éd., Paris, 1868, réimpr. Hildesheim/New York, Georg Olms, 1972 et, plus récemment, Azouvi, Descartes et la France, p. 15-65.

${ }^{23}$ Les ouvrages de philosophie les plus notables du courant novantique en France sont J.-B. Du Hamel, De Consensu veteris et novae philosophiae libri duo, Paris, Savreux, 1663. Du même, Philosophia vetus et nova ad usum scholae accomodata, Paris, 1678. P. Le Gallois, "Parallèle des principes de la physique d'Aristote et de celle de M. Descartes, où l'on montre clairement la conformité des sentiments de ces deux philosophes", in Conversations tirées de l'Académie de M. l'Abbé Bourdelot contenant diverses recherches et observations physiques, Paris, Thomas Moette, 1672, p. 279-348, repris par R. Le Bossu, dans un volume séparé intitulé Parallèle des principes de la physique d'Aristote et de celle de René Descartes, Paris, Le Petit, 1674. Ce courant a encore été peu étudié ; voir cependant M. Grene, "Aristotelico-Cartesian Themes in Natural Philosophy: Some Seventeenth-Century Cases”, Perspectives on Science, vol. 1, n 1, 1993, p. 66-87.

24 Journal des savants, 1666, XXXIV, p. 395.

25 Voir sur ce point l'analyse de C. W. T. Blackwell, “The Case of Honoré Fabri and the Historiography of Sixteenth and Seventeenth Century Jesuit Aristotelianism in Protestant History of Philosophy: Sturm, Morhof and Brucker", Nouvelles de la République des Lettres, 1995, t. XV, 1, p. 49-78, particulièrement p. 54-61. Sur Raey et Heereboord, voir Grene, “AristotelicoCartesian Themes”, op. cit., et T. Verbeck, “Tradition and Novelty: Descartes and some Cartesians”, in The Rise of Modern Philosophy, op. cit., p. 211-222. 
légers que plaisants - c'est bien ce que faisaient alors les jésuites anti-cartésiens, Louis Le Valois, Ignace-Gaston Pardies, René Rapin, Gabriel Daniel. D’où, en second lieu, la représentation de Fabri sur le mode de l'échec : non seulement il n'a pas réussi à rénover l'aristotélisme, mais ses penchants cartésiens le firent persécuter par les siens ${ }^{26}$.

Les alea successifs d'une renommée ne sont pas seulement un miroir de nos vanités : ils révèlent les horizons interprétatifs qui émergent progressivement en histoire des idées, voire en constituent le principal fond de commerce. L'entreprise de Fabri est devenue irrecevable dès la fin du XVII ${ }^{\mathrm{e}}$ siècle en raison à la fois de la polarisation du champ de la philosophie naturelle entre "anciens" et "nouveaux" et de l'assimilation de la nouveauté au cartésianisme. Au XVIII e siècle, Fabri conservera bien une certaine notoriété pour quelques résultats ponctuels et techniques ${ }^{27}$, mais l'affaire était déjà entendue et le temps effacera jusqu'au souvenir qu'il y avait peut-être eu des scolastiques point trop stupides au siècle de Descartes. Symptomatique à cet égard est l'article consacré à Fabri dans la Biographie universelle ancienne et moderne :

\begin{abstract}
Trop tôt distingué et prôné dans le monde savant, sa douceur et sa modestie firent bientôt place à un amour-propre qui étouffa le germe de son talent. Il crut tout savoir parce qu'il avait tout entrepris, sans avoir eu le temps de rien approfondir [...]. Une foule d'écrits sont sortis de sa plume ; mais la plupart sont morts avec les circonstances qui les a faits naître [...] Sa constance à attaquer ou à défendre tout ce qui lui offrait l'occasion de faire quelque bruit lui avait fait donner par quelques auteurs le surnom d'Avocat des causes perdues ${ }^{28}$.
\end{abstract}

Fabri est alors matière à ironie, il ne reste de lui qu'un tempérament et un surnom : pour ses positions théoriques, elles sont tombées dans les oubliettes de l'histoire. Ce sont quelques-unes des dimensions de l'espace dans lequel ces positions théoriques ont été possibles que les paragraphes suivants restitueront.

\footnotetext{
26 J. Brucker, Historia critica philosophiae, 1766, vol. IV, p. 143, cité par Blackwell , "The Case of Honoré Fabri”, op. cit., p. 64 : “[...] nec Scholasticam philosophiam emendavit, et ad salubriora consolades revocare potuit, qui Cartesianismi eum suspectum reddentes [...]". Encyclopédie, Genève, Pellet, 1778, art. "péripatéticienne" 364a-b : "Nous restituerons seulement ici quelques noms moins importants qu'on a omis [dans l'article "aristotélisme”], et qui peut-être ne valent guère la peine qu'on les sorte de l'oubli. [...] François Alphonse, François Gonzales, Thomas Compton, François Rasler, Antonius Poluus, Honoré Fabri : celui-ci, soupçonné dans la société de favoriser le cartésianisme, y souffrit de la persécution".

27 L'Encyclopédie mentionne ainsi la Synopsis optica de Fabri dans les articles "catoptrique" et "parallélisme des rangées d'arbres" ; il en est de même dans l'Histoire des mathématiques de J.-E. Montucla, 4 vol., Paris, H. Agasse, an VII-an X, vol. II, p. 71, p. 304, p. 406, p. 551. Je dois ces renseignements à Pierre Crépel, communication personnelle.

28 Biographie universelle ancienne et moderne, 44 vol., Paris, Madame C. Desplaces et M. Michaud, 2 ème éd., 1855 , vol. XIII, p. 288-289.
} 


\section{Fabri et la science nouvelle : Galilée}

Comme dans un mauvais roman à énigme, on commencera par une fausse piste, d'ailleurs empruntée par les quelques exégètes fabristes existant, parce qu'ils projettent sur les années 1640 ce qui ne sera vrai que vingt ans après. Dans une lettre à Descartes de mars 1646, Mersenne fait l'éloge du jésuite et annonce la publication de ses cours par Mousnier ${ }^{29}$. Descartes a alors une réaction pour lui exceptionnelle : il demande à lire la philosophie de Fabri et s'engage à rendre publique l'opinion qu'il en prendra ${ }^{30}$. C'est qu'il est à l'affût du moindre frémissement des jésuites, espérant qu'ils adopteront le manuel qu'il a publié environ une année plus tôt, les Principia philosophiae : il suppose conséquemment que l'ouvrage annoncé constitue la réaction tant attendue du corps des jésuites. En l'examinant, il défendrait donc ses Principia "contre toute la Société"31.

Lorsque Descartes aura le De Motu entre les mains, il déclarera que Fabri n'a rien établi de solide. Descartes est coutumier des jugements à l'emporte-pièce, mais il faut bien dire qu'il n'avait pas trouvé ce qu'il attendait chez Fabri. A l'époque, ce dernier ne connaissait pas les Principia et, pour lui, Descartes était au mieux l'auteur de la Dioptrique et des Météores $^{32}$. L'homme qu'on retrouve presque à toutes les pages des ouvrages de 1646, l'homme auquel il faut alors se mesurer, bref l'homme à abattre, c'est Galilée, condamné par le Pape en 1633. C'est pourquoi on commencera par analyser la stratégie que Fabri met en œuvre contre Galilée dans le cas particulier du mouvement naturellement accéléré ; c'est seulement ensuite que les thèses de Fabri seront confrontées à celles de Descartes, là encore dans un cas particulier, celui des formes substantielles.

${ }^{29}$ Cette lettre est citée supra note 17.

30 Descartes à Mersenne, 7 septembre 1646, in Correspondance de Mersenne, vol. XIV, p. $443-444$ : "Je serais bien aise de voir [...] la nouvelle philosophie du père Fabri, puisque vous dites qu'on la préfère à la mienne, et qu'elle lui est opposée. Je ne manquerai pas de vous en écrire mon sentiment si tôt que je l'aurai vue et peut-être le ferai imprimer si la chose le mérite". 31 ibid., p. 445. Voir aussi Descartes à Mersenne, 2 novembre 1646, in Correspondance de Mersenne, vol. XIV, p. 585 : “Si le père Fabri n'écrit rien contre moi, je ne me soucie pas aussi de le voir ; mais pour ce qu'on vous avait dit qu'il écrivait toute la philosophie beaucoup mieux et en meilleur ordre que je n'ai fait, je pensais que les jésuites eussent dessein de l'opposer à moi, et en ce cas je serais bien obligé de voir son livre, afin de tâcher de me défendre ; mais rien ne serait cependant si pressé que je ne pusse bien attendre à le recevoir par mer". La réaction de Descartes repose sur l'idée que les Jésuites constituent un corps uni et joint, par ailleurs attestée chez Descartes ; voir par exemple la lettre à Huygens de mars 1636, les Septièmes Réponses et la lettre à Dinet qui les accompagnent, in Oeuvres de Descartes, C. Adam et P. Tannery éd., nouv. prés. par B. Rochot et P. Costabel, 11 vol., Paris, Vrin, 1964-1974, resp. vol. II, p. 52, vol. VII p. 452 et vol. VII p. 564.

${ }^{32}$ La Philosophia, lib. III, cap. 2, art. 2., p. 79, indique quels auteurs il convient de lire sur chaque sujet en physique : Descartes est mentionné seulement à propos de l'arc-en-ciel et de la vision, aux côtés de Christophe Scheiner et de François Aguillon (voir aussi, toujours à propos d'optique, lib. VI, cap. 2, art. 1, p. 119). Dans le même style, la table des matières des Méditationes est accompagnée de la remarque que les objections de Gassendi sont ce qu'il y a de plus beau dans l'ouvrage (Philosophia, lib. IV, cap. 2, art. 3, p. 102). 
Dès août 1643, Fabri soutient que la loi de la chute des corps énoncée par Galilée (selon laquelle les espaces traversés par un corps en chute libre dans des intervalles de temps successifs et égaux sont comme la suite des nombres impairs) est inexacte : d'après lui, les espaces traversés sont comme la suite des nombres naturels ${ }^{33}$. Le fondement de cette loi alternative, tel qu'il est exposé au livre II du De motu, est sommairement le suivant ${ }^{34}$. La notion fondamentale en physique est celle d'impetus : tout mouvement a une cause, l'impetus ${ }^{35}$. Si les corps qui tombent naturellement vers le centre de la terre sont doués d'un mouvement accéléré, c'est que, à chaque instant, il y a production d'un nouvel impetus, et que, d'instant en instant, Dieu conserve les impetus acquis. Pour la détermination quantitative de cettte accélération naturelle, elle dépend de la notion métaphysique d'instant. Comme Fabri l'expliquera dans la Metaphysica, l'instant est un indivisible fini, c'est la portion de temps, plus grande ou plus petite, dans laquelle une action physique prend place ${ }^{36}$. Dans le premier instant de la chute, le corps a donc le temps d'acquérir un premier degré d'impétuosité, dans le deuxième, il en acquiert un deuxième, etc. Puisque les instants sont des grandeurs finies et qu'à chaque instant il y a un nouvel impetus, l'accélération se fait selon la suite des nombres naturels ${ }^{37}$.

Si Fabri s'oppose à Galilée, c'est par conséquent qu'il estime que ce dernier se trompe sur les principes physiques : le temps n'est pas un continu, mais est composé d'instants ; un corps en chute libre ne passe pas par tous les degrés de vitesse, mais acquiert à chaque instant un impetus fini ${ }^{38}$. Il est vrai que

33 Cette loi alternative avait également été proposée par Michel Varro ; voir M. Camerota et M. O. Helbing, All’ Alba della scienza galileana. Michel Varro e il sui De Motu tractatus, Cagliari, Cagliari U.P., 2000. Une liste des lois alternatives à celle de Galilée est donnée in M. Mersenne, Cogitata physico-mathematica, in quibus tam naturae quam artis effectus admirandi certissimis demonstrationibus explicantur, Paris, Antoine Bertier, 1644, p. 49-52.

34 Je suis ici les excellents articles de C.-R. Palmerino, "Infinite Degrees of Speed. Marin Mersenne and the Debate over Galileo's Law of Free Fall", Early Science and Medicine, vol. 4, n 4, 1999, p. 271-328 et "Two Aristotelian Responses to Galilei's Science of Motion: Honoré Fabri and Pierre Le Cazre”, in Jesuit Mathematics and Natural Philosophy in the Early Modern Period, M. Feingold éd., Dortrecht, Kluwer, 2002, p. 181-227.

35 De Motu, lib. I, p. 1 : “Immo ausim dicere ex unius impetus cognitione, non modo motum ipsum, verum etiam totam rem Physicam pendere".

36 Ibid., lib. II, theor. 36, scol., p. 87 : “[...] temporis natura aliter explicari non potest, quam per instantia finita, ut demonstrabimus in Metaphysica ; quicquid sit, voco instans totum illud tempus, quo res aliqua simul producitur, sive sit majus, sive minus”. Metaphysica, lib. IX, prop. 12, p. 371 : “[...] dantur instantia physica, quia datur actio, per quam res est, actio inquam tota simul, et determinata, cum enim res, permanens scilicet, incipiat tota simul, igitur ejus fieri est totum simul [...] dixi etiam determinatam quia cum ita sit, determinata est”. Que des instants puissent être plus ou moins grands contrevient évidemment à l'invariance scalaire que suppose la notion de loi de la nature.

37 De Motu, lib. II, theor. 33-44, p. 87-89.

38 De Motu, lib. II, theor. 127, scol., p. 131 : “[...] si tempus constet ex infinitis actu partibus [...], non potest esse alia progressio, in qua fiat acceleratio motus naturalis, quam illa Galilei juxta hos numeros 1.3.5.7. [...] ; si vero tempus constat ex finitis instantibus aequalibus, nulla datur progressio motus naturaliter accelerati [...] ; si tempus constat ex finitis 
la loi galiléenne semble confirmée expérimentalement : on ne voit pas le corps "sauter" d'une vitesse à l'autre, il accélère continûment ${ }^{39}$. C'est que, répond Fabri, nos expériences sensibles ne concernent pas les véritables indivisibles de temps que sont les instants, mais de gros conglomérats d'instants : de même que, de loin, on ne voit pas les marches d'un escalier, mais un plan incliné, de même, à notre échelle, on ne voit pas du discontinu, mais du continu ${ }^{40}$. C'est pourquoi, selon Fabri, l'évidence expérimentale est impuissante à réfuter la loi selon la suite des nombres naturels ; dès lors, continue-t-il, elle est véritable, puisque par ailleurs conforme à des principes dont la métaphysique a montré la certitude.

Cet arbitrage était préparé dans la Philosophia, traité more geometrico sur la méthode propre à chaque science. Dans le chapitre consacré à la méthode physique, l'exemple, non seulement explicite mais appuyé, du cancre épistémique, c'est Galilée ${ }^{41}$. Fabri soutient en effet qu'on doit réserver le nom d'“expérience physique" à "un effet sensible, certain et évident physiquement"; dès lors ne mérite pas ce nom une proposition comme celle de Galilée : ayant pour objet une accélération et prétendant établir une égalité des temps et des espaces, elle fait intervenir des entités ne tombant pas directement sous les sens ${ }^{42}$. La proposition de Galilée n'est pas non plus un théorème, parce qu'elle ne donne pas le “propter

instantibus actu et infinitis potentia, non potest esse alia progressio hujus accelerationis, quam haec nostra [...] juxta numeros 1.2.3.4.5”. Palmerino, "Two Jesuit Responses”, op. cit., montre que la théorie selon laquelle le temps est composé d'instants égaux et qui, d'après Fabri, interdit toute accélération, est celle qu'avait défendu Arriaga en 1632 dans son Cursus philosophicus.

${ }^{39}$ Ibid., lib. II, Dissertatio, corr. 3, p. 108 : “[...] haec esto clavis hujus difficultatis ; progressio simplex principium physicum habet, non experimentum ; progressio numerorum imparium experimentum non principium”.

40 Ibid. : "Linea denticulata eodem modo se habet physice, hoc est sensibiliter, ac si esset recta". Ibid., lib. II, theor. 127, scol., p. 131 : "Quia illa finita instantia sunt fere innumera in qualiber parte sensibili temporis, in praxi sine sensibile errore in partibus temporis sensibilibus possumus adhibere progressionem Galilei”. Fabri à Mersenne, 21 août 1643, in Correspondance de Mersenne, vol. XII, p. 289-290 : "D'autant plus grande je prends la partie de temps, j’ai toujours trouvé que la différence de Galilée et de la mienne était moindre en comparaison de tout l'espace [...]. Or est-il que cette différence est toujours moindre, puisque la moindre partie de temps qu'on puisse prendre contient pour le moins 1.000 .000 instants".

${ }^{41}$ Philosophia, lib. III, cap. 2, art. 2 et lib. III, cap. 4, art. 2, resp. p. 80 et p. 88-89. Ce dernier article est commenté in P. Dear, Discipline and Experience. The Mathematical Way in the Scientific Revolution, Chicago, Chicago U.P., 1995, p. 138-143. Dans le même esprit, Fabri affirme avoir accompli l'expérience de Torricelli à Lyon au début des années 1640, mais que celleci ne prouve pas à proprement parler le vide (Metaphysica, Appendix 1, p. 570 ; Grassi à Baliani, 26 décembre 1647, in Correspondance de Mersenne, vol. XV, p. 593-594).

42 Philosophia, lib. III, cap. 4, art. 2, §§ 1-2, p. 88 : “Experimentum physicum, est effectus aliquis sensibilis, certus et evidens physice, id est, ita ut citra miraculum fallere non possit, v.g., video lapidem modo moveri, modo non moveri ; idem video in globo plumbeo, ligneo ; sentio majorem ictum lapidem ex majori altitudine cadentis, etc. Porro experimenti nomine carere debet, id omne quod in sensum non cadit, v.g., aequalitas duorum temporum [...] ; vel aequalitas spatiorum [...]”. Ibid. § 5 : "Hypothesis physica, est propositio de aliquo effectu asseritur quod sit, eo quod omnia experimenta consentiant". A ce compte, la loi de Fabri est également une hypothèse puisqu'elle repose, comme on vient de le voir, sur la notion métaphysique 
quid sit", mais seulement le "quod sit"43. Elle ne mérite même pas le nom d'"hypothèse", étant liée à une expérience qui n'est pas telle que toute autre hypothèse soit inconciliable avec elle ${ }^{44}$.

Fabri n'a pas mis en place ces distinctions épistémiques à l'occasion de son combat contre Galilée. La primauté des phénomènes sensibles est une caractéristique constante de ses écrits physiques, et il pensait pouvoir établir une physique démonstrative fondée sur une analyse des qualités. En mettant ses options épistémiques au service de ce combat, il se conforme cependant à la stratégie en deux points que la Compagnie de Jésus avait mise en place : attaquer la science galiléenne tous azimuts, y compris sur des points qui ne sont pas logiquement liés au copernicanisme ; ne pas contester le paradigme galiléen, mais montrer que, dans ce paradigme, on peut faire mieux que Galilée. Mais, à partir du moment où Fabri est mesurée à l'aune de son orthodoxie, il est remarquable que son combat anti-galiléen soit allé de pair avec la défense de la proposition que des grandeurs continues sont composées d'un nombre fini d'indivisibles étendus ${ }^{45}$. Cette proposition a en effet été l'objet de censures à répétition dans la compagnie de Jésus et sera officiellement condamnée dans l'Ordinatio pro Studiis superioribus de $1651^{46}$. On a là un cas curieux de l'art d'écrire : en arriver à soutenir une proposition totalement hétérodoxe pour dévaloriser un auteur condamné par les autorités.

\section{Fabri et la philosophie nouvelle : Descartes}

Comme on l'a fait remarquer plus haut, la Physica publiée en 1669-1671 vient accomplir le projet éditorial entamé en 1646. Cela n'empêche pas que l'intention de Fabri ait totalement changé : cette fois, et conformément à l'humeur du moment, l'adversaire est bien Descartes. Les allusions sont transparentes dès les premiers paragraphes, où Fabri attaque les recentiores qui confondent la physique et la géométrie,

d'instant. Sur l'introduction d'hypothèses dans la physique du XVII ${ }^{\mathrm{e}}$ siècle, voir S. Roux, "Le scepticisme et les hypothèses de la physique", Revue de synthèse, $4^{\mathrm{e}} \mathrm{s}$., $\mathrm{n}^{\mathrm{OS}}$ 2-3, avril-septembre 1998, p. 211-255.

43 Ibid., § 7, p. 89.

44 Ibid., § 10 : "Itaque haec esto regula certissima. Hypothesis physica ex certo tantum experimento sequitur, cum quo necessariam connexionem habeat ; id est quae certa sit, dum illud sit certum".

45 Fabri présente sommairement sa notion du continu dans la lettre à Mersenne, 21 août 1643, Correspondance de Mersenne, vol. XII, p. 290-291 : "Je réponds $1^{\circ}$ que pouvant expliquer facilement tout le continu avec des points, je ne dois pas y mettre des parties infinies si les finies sont suffisantes ; $2^{\circ}$ que cette infinité de parties actuelles est inimginable et peut être détruite par de très évidentes démonstrations ; $3^{\circ}$ je dis pour expliquer brièvement ce que j'en pense, et j'en pense à mon avis ce qui est, que le continu, une ligne par exemple, n'est pas divisible actuellement jusques à l'infini, mis seulement en puissance”.

46 L'Ordinatio est une liste des propositions interdites d'enseignement ; elle fut rédigée par une commission dirigée par le général des jésuites de l'époque, Francesco Piccolomini, à la demande de la neuvième Congrégation générales. Les propositions interdites sur les indivisibles se trouvent in Ratio Studiorum et institutiones scholasticae Societatis Jesu, 3 vol., Berlin, A. Hoffmann \& Comp., 1887-1894, vol. III p. 92. Palmerino, “Two Jesuit Responses”, op. cit., montre de surcroît que les indivisibles que Fabri supposa finalement pour répondre aux critiques suscitées par les ouvrages de 1646-1648 sont susceptibles de contraction et d'expansion, ce qui était encore pire du point de vue de l'orthodoxie. 
forgent à loisir des hypothèses et fabriquent un nouveau monde. Procéder de la sorte, c'est être aussi ridicule que Démocrite, qui, pour mieux comprendre les choses corporelles, qu'on peut voir et sentir, avait cru qu'il devait s'arracher les yeux ${ }^{47}$. Cette critique épistémique du cartésianisme est à l'époque un lieu commun chez ceux qui contestent, pas forcément de manière rétrograde, la grandeur cartésienne : Descartes eut l'esprit indûment géométrique, il fut dogmatique, indifférent aux questions de fait, confiné dans ses spéculations et ignorant de l'empirie ; sa physique n'est qu'un roman ${ }^{48}$. Il n'est dès lors pas étonnant que Fabri critique ce qui passe alors pour pierre de touche de la physique cartésienne - par exemple, le recours à la matière subtile pour expliquer les phénomènes ou les lois du mouvement que l'on commence à savoir fausses.

Pourtant, il entend conserver un certain nombre d'acquis de la philosophie nouvelle, en particulier l'élimination des formes substantielles. Les traités de 1667 comme la Physica affirment que, si les plantes et les animaux ont des formes substantielles, ces dernières sont, non quelque chose d'absolu, mais de respectif : elles ne sont pas produites par une action spécifique, mais résultent de l'arrangement, de la disposition et de l'organisation de parties matérielles ${ }^{49}$. Accusé par Pardies de cartésianisme, il ne se

47 Physica, “Auctor lectori”, § 3, n.p. Dans les ouvrages tardifs de Fabri, Descartes apparaît constamment comme le chef des démocritéens ; voir par exemple Societatis Jesu ad Patrem Ignatium Gastonem Pardesium ejusdem Societatis Epistolae tres de sua Hypothesi philosophica, Mayence, Petrum Zubrodt, 1674 (par la suite Epistolae tres), § 30, p. 61. Comparationum Tractatus, "Comparatio secunda inter philosophiam peripateticam et democriticam", in Oeuvres de Fabri, vol. IV, 209r-209v. 48 L'assimilation de la physique cartésienne à un roman trouve son origine dans le propos de Pascal rapporté par Menjot: “Feu M. Pascal appelait la philosophie cartésienne le roman de la nature, semblable à peu près à l'histoire de Don Quichotte”. Voir ensuite Chapelain à Carrel de Sainte-Garde, 16 février 1662, in Lettres de Jean Chapelain, P. Tamizey de Larroque éd., 2 vol., Paris, Imprimerie Nationale, 1880-1883, vol. II p. 203-204. Leibniz à Malebranche, mai-juin 1679, in Leibniz. Oeuvres choisies, L. Prenant éd., Paris, Aubier-Montaigne, 1972, p. 140. Huygens, "Compte-rendu de la Vie de M. Des Cartes par Baillet”, in Euvres de Huygens, vol. X, p. 403. C. Perrault, Parallèle des anciens et des modernes en ce qui regarde les arts et les sciences, Paris, J.-B. Coignard, 1692, 2ème éd., vol. I p. 47.

49 Tractatus duo, lib. I, prop. 28, p. 19-21 : "Forma plantae non est ulla entitas absoluta. Haec propositio est contra communem recentiorum scholasticorum sententiam. Non existimo tamen esse contrariam Aristoteli, vel antiquioribus philosophie, et verissimam esse contendo [...] Forma plantae nihil absolutum est, sed resultat ex tali plexu, dispositione, organisatione [...] Omnis forma praeter animam rationalem est aliquid respectivum. [...] Forma plantae etiam divinitus extra plantam existere non potest'. On trouve des énoncés analogues à propos de la forme sensitive des animaux au lib. V, prop. 66, p. 164-165. Voir de même Physica, tract. V, lib. I, prop. 65, vol. III, p. 41 : “Omnis forma praeter animam rationalem dat esse respectivum : v. gr. esse lignum est esse respectivum, quia resultat ex tali scilicet elementorum plexu; ex hoc enim resultat tale complexum proprietatum sensibilium, v. gr. esse durum, fissile, inflammabile, etc. sed profecto hoc esse est respectivum, quod scilicet sine nova actione propria resultat : in elementis, res est clarissima, v. gr. ignis esse in eo consistit, quod sit lucidus et calidus in summo ; igitur quod multa vi, tum calefaciaendi, tum lucendi polleat; atqui haec vis ex collectione multarum partium resultat ; in mixtio res etiam non est minus perspicua, in plantis aeque facilis est [...] ; in brutis animalibus major difficultas esse videtur, sed eam quoque, ni fallor, suo loco componabimus, faciemusque, ut constet animalis puri, hoc 
rétractera pas : la seule forme substantielle absolue est l'âme raisonnable ; prenant le bois pour exemple, il reconnaît qu'il y a une différence entre sa matière et sa forme substantielle, mais, précise-t-il, de raison seulement puisque "la forme substantielle est seulement le rapport, la raison, l'espèce, l'acte, le logos, résultant de cette composition des éléments et des autres miscibilia"50. Autrement dit, la forme substantielle du bois n'est pas un être différent en réalité de ses parties matérielles, mais seulement un nom qui désigne leur rapport ; en particulier, elle n'existe pas à titre séparé. Il n'est que de connaître quelques énoncés de cartésiens notoires comme Gérauld de Cordemoy ou Antoine Arnauld pour comprendre que les positions de Fabri sur les formes substantielles aient pu être taxées de cartésiennes ${ }^{51}$.

A ce point, on pourra s'étonner. L'élimination des formes substantielles a été un des articles du cartésianisme condamné par les pouvoirs politiques et religieux à partir des années $1660^{52}$. Fabri a joué

est, quod sit tantum principium sentiendi, esse respectivum, ac resultare per generationem, sive ulla actione propria [...]”. Ou encore ibid., tract. VI, lib. I, prop. 21, vol. III, p. 224 : "Forma mixti est aliquid respectivum nec enim est entitas quaedam absoluta, ut multi e Scholasticis opinantur : nam etiam illa saltem per miraculum destructa, manente dumtaxat eadem elementorum mixtione, eodem plexu, etc., maneret adhuc esse mixti ; igitur forma mixti ; igitur esset et non esset, quod fieri non potest ; ergo non est entitas quaedam absoluta, est tamen aliquid, quod enim nihil est nullum esse rei dar potest, igitur est aliquid respectivum ; analogiam habes in artefacto, cujus forma ex tali partium positione et situ resultat, vel in consonatia diapason". Je n'ai pas trouvé de formule équivalente dans les traités de 1646-1648, mais les thèses que Fabri fit à l'époque soutenir, citées supra note 3, montrent que ce point était alors acquis au moins en ce qui concerne les plantes.

50 Epistolae tres, p. 53 : “forma substantialis est merus respectus, ratio, species, actus, logos, resultans ex talis compositione elementorum, aliorumque miscibilium". Selon la théorie aristotélicienne des mixtes, les miscibilia sont les parties qui s'unissent pour composer un véritable mélange, c'est-à-dire un mélange dont toute partie est de même nature que le tout. Sur la théorie des mixtes chez Fabri, voir D. Des Chene, "Wine and Water: Honoré Fabri on Mixtures", in C. H. Lüthy, I. E. Murdoch, W. R. Newman éd., Later Medieval and Early Modern Corpuscular Matter Theories, Leide, Brill, 2001, p. 363380. Des extraits des Epistolae tres sont donnés par P. R. Blum, "Aristotelianism more geometrico: Honoré Fabri”, in Blackwell et Kusukawa éd., Philosophy in the Sixteenth and Seventeenth Centuries, op. cit., p. 234-247.

51 “Ce n'est pas que ces mots [qualité et forme] ne soient propres à exprimer le différent arrangement des parties de la matière, mais ils ne peuvent raisonnablement signifier autre chose" (Six Discours sur la distinction et l'union du corps et de l'âme, Premier discours, in Euvres philosophiques, P. Clair et F. Girbal éd., p. 105). "La forme est ce qui rend une chose telle, et la distingue des autres, soit que ce soit un être réellement distingué de la matière, selon l'opinion de l'Ecole, soit que ce soit seulement l'arrangement des parties" (La Logique ou l'art de penser, Paris, Flammarion, 1970, liv. III, chap. 18, p. 302).

52 Les principaux faits et documents se trouvent in V. Cousin, Fragments philosophiques pour servir à l'histoire de la philosophie, Paris, 1866, 2ème éd., réimp. Genève, Slatkine, 1970, t. III p. 297-332. Bouillier, Histoire de la philosophie cartésienne, op. cit., vol. I, p. 466-485. R. Ariew, "Quelques condamnations du cartésianisme : 1662-1706”, Bulletin cartésien XXII, Archives de philosophie, 57, p. 1-6. Pour des interprétations récentes, voir en premier lieu T. McClaughin, “Censorship and Defenders of the Cartesian Faith in Mid-Seventeenth Century France”, Journal of the History of Ideas, oct-déc. 1979, vol. $40, \mathrm{n}^{\circ} 4$, p. 563-581. Ariew, Descartes and the Last Scholastics, op. cit., particulièrement p. 140-187. Azouvi, Descartes et la France, op. cit., p. 15-47. 
dans ces condamnations un rôle non négligeable, pour ne pas dire décisif : le 15 avril 1660, il censure privatim l'explication physique de la transsubstantiation qu'avait proposée Descartes ; il a contribué à la mise à l'index des œuvres de Descartes donec corrigantur le 20 novembre 166353. Comment donc concilier les positions de Fabri sur les formes substantielles, sa contribution à la condamnation des œuvres cartésiennes et le fait qu'un des articles condamnés soit l'élimination des formes substantielles ? Des tireurs d'élite du parti cartésien comme Antoine Arnauld ou Jacques Rohault auront d'ailleurs beau jeu de souligner la contradiction : il n'est pas logique de condamner Descartes pour avoir éliminé les formes substantielles alors que Fabri, dans un ouvrage dédié au général des Jésuites, les rejette tout aussi bien $^{54}$. La mise à plat du problème - sinon la résolution de la contradiction - demande une bonne dose de charité et une analyse un peu plus détaillée des chefs d'accusation du cartésianisme dans les années 1660-1680.

Pour le dire d'un mot, l'élimination des formes substantielles constituait pour Fabri un acte théorique isolé, nullement incompatible avec sa condamnation de deux thèses cartésiennes qu'il estimait incompatibles avec le dogme de la transsubstantiation : la thèse que l'étendue est l'essence des corps et la thèse que les qualités que nous percevons dans les corps ne leur appartiennent pas réellement ${ }^{55}$. D'après ce dogme en effet, le corps de Jésus-Christ est tout entier dans l'eucharistie et les espèces du pain et du vin sont des accidents "réels" ou "distincts" 56 . A ce point, l'erreur des commentateurs est de supposer

53 Les lettres de Descartes sur la transsubstantiation avaient été transmises à Fabri par Bertet, qui lui-même les tenait de Clerselier ; la censure de Fabri est publiée in G. Sortais, "Le Cartésianisme chez les Jésuites français au XVII et au XVIII siècle”, Archives de philosophie, 1929, vol. VI, cah. 3, p. 51. Baillet, La Vie de M. Descartes, op. cit., liv. VIII, chap. 9, vol. II, p. 529, affirme que la mise à l'index a résulté des "intrigues d'un auteur particulier", qu'il identifie en marge comme "P. H. Fabri”. La signification du donec corrigantur n'est pas établie : dans la mesure où il n'y a aucune précision sur les corrections à faire, pour Baillet, on peut continuer à lire Descartes comme si de rien n'était ; pour Arnauld, "c'est la même chose que si un livre était condamné absolument” (A M. du Vaucel, 19 octobre 1691, Oeuvres, 43 vol., Lausanne/Paris, Sigismond d'Arnay \& Co, 1775, vol. III, p. 398).

54 Arnauld, Plusieurs raisons pour empêcher la censure et la condamnation de la philosophie de Descartes, [Paris, 1671], in V. Cousin, Fragments philosophiques, p. 303-317. Rohault, Entretiens sur la philosophie par M. Rohault, [s.n], 1672, p. 3031. De manière non polémique, la position conciliatrice de Fabri est aussi relevée par Leibniz, Hypothesis physica nova seu Theoria motus concreti, §56, in Leibniz. Sämtliche Schriften und Briefe, Preussische (puis Deutsche) Akademie der Wissenschaften éd., Darmstadt (puis Leipzig puis Berlin), 1923- (par la suite Leibniz. Sämtliche Schriften), VI 2, p. 247. Ils se réfèrent tous trois aux énoncés cités supra note 49.

55 La possibilité de la transsubstantiation est analysée dans la Metaphysica, V, prop. 51-prop. 85, p. 184-194, mais le lien avec les thèses des "nouveaux démocritéens" apparaît principalement dans les manuscrits de Fabri ; voir par exemple "De l'eucharistie", chap. 3, in Oeuvres de Fabri, vol. IV, particulièrement f. 102 sqq. Dialogi theologici, Dialogus quartus, De Speciebus Eucharistis, in Oeuvres de Fabri, vol. II, f. 102-157.

56 Pour des mises au point sur la transsubstantiation au XVII ${ }^{\mathrm{e}}$ siècle, voir J.-R. Armogathe, Theologia cartesiana. L'explication physique de l'eucharistie chez Descartes et Dom Robert Desgabets, La Haye, Nijhoff, 1977. P. Redondi, 
qu'une ontologie systématique comme l'était l'ontologie aristotélicienne "faisait bloc", et qu'il n'était pas possible de ne pas rejeter les accidents réels une fois qu'on avait éliminé les formes substantielles. (Erreur d'ailleurs bien naturelle : les accidents distincts et les formes substantielles sont les deux faces d'une même pièce, et ce que le dogme exige, c'est que les uns et les autres puissent surnaturellement avoir une existence séparée.) En fait, Fabri a jugé bien au contraire, peut-être sous la pression des circonstances et en tout cas pas très logiquement, que les deux questions pouvaient être soumises à un traitement différencié : pour lui, l'élimination des formes substantielles est une exigence philosophique (elles introduisent des problèmes et n'en résolvent aucun), la distinction du corps et de l'étendue et la conservation des accidents réels, des nécessités théologiques (si le corps du Christ n'est pas distinct de son étendue, il est impossible qu'il soit dans une petite étendue comme celle de l'eucharistie et, plus encore, qu'il soit dans plusieurs hosties en même temps ; si les accidents ne peuvent pas subsister indépendamment de leur substance, le pain n'a pas été transformé en corps du Christ dès lors qu'on voit encore les apparences du pain). A cette interprétation, on pourrait opposer que les anti-cartésiens des années 1660-1680 s'opposent aussi à l'élimination des formes substantielles, et lient par là-même le destin des différentes entités scolastiques. Mais précisément, à ma connaissance, la question des formes substantielles est toujours distinguée des questions des accidents réels et de la nature de l'étendue, et contrairement à ces dernières, elle n'est jamais liée au dogme de la transsubstantiation : lorsque le maintien des formes substantielles est l'objet d'une argumentation explicitement liée à la foi, celle-ci consiste simplement en ceci que le privilège accordée à l'âme humaine ne durera pas longtemps une fois toutes les autres formes substantielles éliminées ${ }^{57}$.

Galilée hérétique, trad. M. Aymard, Paris, Gallimard, 1985. A. Del Prete, texte introductif à l'édition des “Explications sur le mystère de l'Eucharistie suivant les principes de la philosophie de Descartes”, La Lettre clandestine, 10, 2001, p. $226-260$.

57 Mon interprétation repose sur la lecture des différentes condamnations reproduites in Ariew, "Quelques condamnations du cartésianisme", op. cit., ainsi que des ouvrages suivants : R. J., [A. Rochon], Lettre d'un philosophe à un cartésien de ses amis, , Paris, Thomas Jolly, 1672, §§ 2-6 et $\S \S 26-31$, resp. p. 4-12 et p. 66-81. J.-B. La Grange, Les Principes contre les nouveaux philosophes Descartes, Rohault, Gassendi, le père Maignan, etc., Paris, Georges Josse, 1675, chap. 6-chap. 8, p. 99135. F. Babin, Journal ou relation fidèle de tout ce qui s'est passé dans l'université d'Angers au sujet de la philosophie de Des Chartes en l'exécution des ordres du Roy pendant les années 1675, 1676, 1677 et 1678, Angers, 1679, p. 39, p. 41. L. de La Ville [Le Valois], Sentiments de M. Descartes touchant l'essence et les propriétés des corps, opposés à la doctrine de l'Eglise, et conforme aux erreurs de Calvin, sur le sujet de l'Eucharistie, Paris, Etienne Michallet, 1680, Seconde partie, chap. 1-2, p. 99-112. [anonyme], La Philosophie de Descartes contraire à la foi catholique, Paris, Guy Caillou, 1682. Elle est confortée par les documents publiés par D. Kahn, "La Condamnation des thèses d'Antoine de Villon et Etienne de Clave contre Aristote, Paracelse et les cabalistes (1624)", in Revue d'Histoire des sciences, tome 55-2, 2002, p. 143-198 et par S. Matton, "Note sur quelques critiques oubliées de l'atomisme : A propos de la transsubstantiation eucharistique”, in ibid., p. 287-294. Dans ses Entretiens sur la philosophiee, op. cit., p. 32, Rohault, qui consacre par ailleurs une soixantaine de pages à la question des accidents réels, discrédite d'une ligne l'affirmation de M. N. selon laquelle la transsubstantiation exigerait des formes substantielles, en remarquant que M. N. est incapable de préciser à quel concile il se réfère. Il resterait à rendre compte, 


\section{Fabri et les anciens : Aristote contre les scolastiques}

Fabri eut très tôt le sentiment que les savoirs scolastiques, et en particulier la physique, étaient trop décrépits pour n'avoir pas besoin d'un ravalement complet ${ }^{58}$. Ce ravalement, il ne le concevait pas comme une révolution, mais plutôt comme une remise en ordre formelle et comme un retour à la pensée d'Aristote. Remise en ordre formelle tout d'abord : il visait une concaténation more geometrico de propositions fondées sur des classes de phénomènes comme le lourd et le léger, le tendu et le comprimé, le sec et l'humide, etc. Telle entreprise s'opposait aux commentaires et aux manuels traditionnels, qui, ayant comme principe ordonnateur la suite textuelle des ouvrages aristotéliciens, privilégient l'ordre des mots par rapport à l'ordre des choses ; elle s'opposait tout aussi bien aux nouveaux recueils d'expériences, qui, à force de prôner les faits, renonçaient en fait à toute visée proprement démonstrative ${ }^{59}$. Pour Fabri, il s'agissait de prendre comme point de départ des qualités concrètes et néanmoins pouvant, en raison de leur universalité, donner lieu à des démonstrations ; cette "poïographie", pour parler leibnizien, est incontestablement une des raisons de l'intérêt que Leibniz porta à Fabri ${ }^{60}$.

Fabri entendait également fermer la parenthèse scolastique et restituer la pensée d'Aristote dans son authenticité. La Philosophia dresse contre les scolastiques un réquisitoire aussi cruel que classique : ils ont abusé d'inventions terminologiques, de distinctions subtiles, d'entités barbares ; ils ont voulu réduire la physique à la métaphysique, la métaphysique, à la logique, la logique, à la grammaire ; ignorant la

autrement que par l'opportunisme, de l'ensemble de lettres où Leibniz cherche à promouvoir, auprès de ses correspondants catholiques, sa propre réhabilitation des formes substantielles comme indispensable à la transsubstantiation (voir, par exemple, A de la Chaize, mai 1680, in Leibniz. Sämtliche Schriften, II 1, p. 512).

58 Oeuvres de Fabri, vol. III, f. 78 : "De Physica mea nihil dicam, cum res per se ipsa loquatur ; quid enim physica in scholis habuimus? Absque pudore dici a me vix potest [...]. Nemo ante me demonstrationes exploravit in Physica".

59 Physica, “Auctor lectori”, §§ 4-6, n.p. L’ouvrage déjà cité de P. Dear, Discipline and Experience, porte précisément sur la question de savoir comment des récits d'expériences singulières ont pu, au XVII ${ }^{\mathrm{e}}$ siècle, être intégrées à une physique prétendant à l'universalité et la nécessité.

60 Philosophia, lib. III, cap. 3, art. 1, §§ 3-4, p. 81-82. Alors qu'il rédige l'Hypothesis physica nova seu Theoria motus concreti, Leibniz lit la Physica de Fabri, dont il avait eu connaissance par Oldenburg ; ses notes d'automne 1670 à début 1672 sont retranscrites in Leibniz. Sämtliche Schriften, VI 2, p. 186-218. Fabri sera d'ailleurs un des premiers à réagir à l'Hypothesis (Fabri à Leibniz, 14 novembre 1671, in ibid. II 1, p. 185-188). Quelques années après, Fabri l'ayant classé parmi les imitateurs de Descartes, Leibniz précise ses positions et lui adresse un remaniement de son ancien traité (Leibniz à Fabri, fin 1676, in ibid., II 1, p. 286-301). Ce remaniement est l'aboutissement de tentatives tout à fait similaires à celles de Fabri, visant à constituer une physique démonstrative et non hypothétique, où les phénomènes sensibles les plus connus doivent permettre de déduire les moins connus (voir en particulier le Specimen hypotheseos demonstrativa, in ibid., VI 3, p. 3-10, et la série des Propositiones quaedam physicae, in ibid., VI 3, p. 11-68). Selon E. Caruso, "Honoré Fabri gesuita et scienzato", in Miscellanea secentesca, Milan, Cisalpino, 1987, particulièrement p. 116-126, Fabri a joué un rôle décisif pour la constitution du projet leibnizien de réforme de la logique aristotélicienne. 
puissance de l'arithmétique, de la géométrie et plus généralement de la mathesis, ils ont préféré la dispute au raisonnement ${ }^{61}$. En ce sens, surenchérira la Physica, les scolastiques sont aussi éloignés de la pensée aristotélicienne que le sont les ennemis déclarés d'Aristote, les démocritéens et les épicuriens. Chez les uns comme chez les autres, la prolifération ontologique est d'ailleurs signe de fausseté : la diversification des corpuscules modernes ne vaut pas mieux que la multiplication des entités scolastiques ${ }^{62}$. Les vrais coupables cependant, ce ne sont pas les scolastiques, mais bien les Arabes, "hommes assurément ingénieux, subtils, fins, pénétrants, mais aussi perfides, trompeurs et impies", qui leur ont transmis un texte adultéré, corrompu, perverti63. A ce point, le lecteur un peu averti aura compris que les scolastiques en question sont les scotistes ${ }^{64}$. Pour échapper à leurs arguties, la solution consiste selon Fabri à revenir à la littéralité du texte aristotélicien, à son sens propre et premier ${ }^{65}$. En ce sens, l'éloge qu'il fait de la liberté de philosopher ne doit pas être lu au prisme des Lumières : comme pour d'autres aux XVI et XVII siècles, il ne s'agissait pas d'affirmer le droit de tout individu à penser par lui-même, mais de défendre la lecture des œuvres anciennes contre la prétention à ériger $a b$ ovo une vérité absolument nouvelle et l'Aristote "latin" de Thomas contre l'Aristote "arabe" de Duns Scott ${ }^{66}$.

La rénovation de l'aristotélisme jouait donc pour Fabri à la fois contre les "nouveaux philosophes" et contre les "anciens scolastiques" - c'est là une de ses autres rencontres avec Leibniz, qui finira d'ailleurs par conclure à l'échec de Fabri67. Dans la "Comparatio quinta Antiquitatis et Novitatis", écrite

61 Philosophia, lib. II, cap. 2, art. 2 ; lib. II, cap. 5, art. 1 ; lib. III, cap. 5, art. 1-2, resp. p. 55-56, p. 65-66, p. 92-93.

62 Physica, “Auctor lectori”, § 41, n.p. Comparationum Tractatus, "Comparatio secunda philosophiam peripateticam inter et democriticam", in Oeuvres de Fabri, vol. IV, f. 208.

63 Physica, “Auctor lectori”, $\S \S 7-8$, n.p. La dissociation entre Aristote et des scolastiques corrompus par les Arabes se trouve aussi chez R. Rapin, Réflexions sur la philosophie ancienne et moderne et sur l'usage qu'on doit en faire pour la Religion (1684), "De la philosophie en général” § 15, in Oeuvres du Père Rapin, 3 vol., La Haye, Pierre Gosse, 1725, t. II p. $357-358$.

${ }^{64}$ L'identification est explicite au moins dans certains manuscrits de Fabri. Roger Ariew travaille depuis quelques années avec l'hypothèse que les scolastiques français du XVII ${ }^{\mathrm{e}}$ siècle auraient été particulièrement marqués par le scotisme.

65 Physica, “Auctor lectori”, § 9, n.p. : “Ego igitur hanc Provinciam suscepi, ut veram ac Germanm doctrinam Aristotelis (...), tum a Democritis, tum ab Arabibus eorumque sequacibus vindicarem". Dans la lettre à Leibniz du 14 nov. 1671 , Leibniz. Sämtliche Schriften, II 1, p. 187, Fabri déclare sa physique tirée "ex simplicissimis principiis, iisque peripateticis ad literam". Epistolae tres, p. 70 : “Totus sum in commendando, laudando et praedicando Aristotelem [...] juxta nativum et proprium literae, textus, et verborum sensus". Comparationum Tractatus, "Comparatio secunda inter Philosophiam peripateticam et democriticam", in Oeuvres de Fabri, vol. IV, f. 210r : "Hoc igitur primum officio defuncti, erga Philosophum, ejusque textibus propris ac vero sensui restitutis, pauca quaedam illius vel explicamus ; vel omnino tollimus".

66 Epistolae tres, p. 57 : "Sed inquiunt [scholastici], Aristotelem in alio sensu intellegimus ; bene est ; gaudeant suo sensu ; cur meo aeque gaudere mihi non licet ?". Ibid., p. 71 : “me liberum esse profiteor ; et veritatem ubique diligo ; sive illam in Aristotele, sive in Epicuro explorem ; et nullo quantum ad hoc factionis studio ducor".

${ }^{67}$ Leibniz à Des Bosses, 2 février 1706, in C. Frémont, L’Etre et la relation, Paris, Vrin, 1981, p. 75 : “Honoré Fabry [...] a trop et cependant trop peu concédé aux Modernes, ou trop et trop peu accordé à l'Ecole. Il a livré à l'ennemi le centre de la 
à la fin de sa vie et à l'époque où la Querelle des Anciens et des Modernes faisait rage, Fabri tente un partage entre les nouveautés qu'on doit condamner, parce qu'elles sont dangereuses pour les particuliers comme pour le public, et les nouveautés qu'il faut non seulement admettre mais louer ${ }^{68}$. On ne s'étonnera pas de trouver dans la première catégorie les innovations touchant la religion, la politique, les régimes de vie et les pratiques médicales (l'idée étant que l'expérimentation en ces matières est le plus souvent dommageable à la santé). La seconde catégorie regroupe pour ainsi dire toutes les innovations dont se vantait Fabri : avoir proposé de nouvelles démonstrations fondées sur les indivisibles de Cavalieri, avoir fait des expériences nouvelles, avoir tiré des conclusions légitimes du corpus aristotélicien, l'avoir mis en ordre géométrique, avoir refusé les entités occultes des aristotéliciens, avoir proposé une exposition claire de tout le système philosophique et religieux ${ }^{69}$. Cela montre, à tout le moins, qu'il eut un bref instant conscience de la difficulté de sa situation historique.

\section{Conclusion : Fabri aujourd'hui ?}

Attirer l'attention sur un personnage comme Fabri, c'est faire œuvre de mémoire. Celui qu'aucune vocation d'antiquaire ne pousse à révérer le passé, pour ainsi dire à la mesure de son obscurité, se demandera cependant à quel titre Fabri peut être considéré comme un objet mémorable.

Certains historiens ont soutenu que Fabri avait indirectement participé à la grandeur de Galilée ou de Descartes, de Huygens, de Leibniz ou de Newton ${ }^{70}$. Ne leur a-t-il pas adressé des objections, comme sur un pied d'égalité ? N'a-t-il pas, comme Galilée, souligné l'importance des expériences et des mathématiques pour la nouvelle science du mouvement ? Sa loi de la chute des corps n'a-t-elle pas été discutée comme une alternative plausible ? N'a-t-il pas, si on l'en croit, enseigné la circulation du sang avant Harvey et fait l'expérience de Torricelli avant Torricelli ? N'a-t-il pas vu les apories du mécanisme cartésien ? N'a-il pas entrepris de rénover more geometrico la logique aristotélicienne ? N'a-t-il pas polémiqué avec Huygens sur les anneaux de Saturne ? N'a-t-il pas, réinterprétant dynamiquement la notion d'indivisible de Bonaventura Cavalieri, anticipé la notion newtonienne de fluxus ? Hélas. La grandeur des grands hommes ne se communique pas si facilement : il y a bien un tribunal de l'histoire, nous ne pouvons lire Fabri aujourd'hui comme nous lisons Torricelli ou Galilée, Huygens ou Descartes,

forteresse péripatéticienne, qu'il devait par dessus tout protéger - je veux dire les âmes des bêtes et les entéléchies [...] pendant qu'il donnait tout son soin à soutenir les fortifications extérieures, situées loin à l'écart, et qui n'offraient rien pour leur défense - je veux dire certaines qualités occultes qu'il a admises comme primitives, par exemple la lourdeur et la tension".

68 Sur ce type de partage et ses implications, voir C. Ginzburg, "High and Low: the Theme of Forbidden Knowledge in the 16th and 17th centuries", Past and Present, 73, p. 28-41.

69 Comparationum Tractatus, "Comparatio Quinta Antiquitatis et Novitatis”, in Oeuvres de Fabri, vol. IV, f. 227v-228v.

70 Je pense ici principalement à E. A. Fellman, “Die mathematischen Werke von Honoratus Fabry”, Physics, 1959,1 et 2, p. 6-25 et p. 69-102 et à A. Boehm, "Deux essais de renouvellement de la scolastique au XVII siècle. II : L'aristotélisme d'Honoré Fabri”, Revue des sciences religieuses, oct. 1965, vol. 39, n 4, p. 305-360. 
Leibniz ou Newton. Fabri n'a pas effectué une synthèse effective qui aurait ouvert de nouvelles perspectives, il n'a pas su opérer autrement que par polémiques successives, se gardant à droite et à gauche, devant et derrière, en haut et en bas. Dès lors, ce n'est pas la grandeur de Fabri qui en fait un objet digne de mémoire ; bien au contraire, et sans aucun paradoxe, seule sa petitesse peut nous intéresser. C'est qu'elle nous fait comprendre à l'issue de quels combats la grandeur de nos grands hommes s'est constituée en héritage, sinon immuable, du moins transmissible.

L'autre intérêt pour nous de Fabri concerne le partage que nous faisons entre orthodoxie et hétérodoxie. Nous avons beau avoir lu Foucault, nous absolutisons toujours un peu trop la norme aux dépens des pratiques, nous projetons toujours un peu trop dans les faits la séparation du savoir et du pouvoir, nous nous imaginons toujours un peu trop que ce dernier émane d'un unique centre. Fabri était membre d'un ordre religieux revendiquant l'“uniformité et la solidité de la doctrine"71, il a nagé dans les eaux troubles et mouvantes du pouvoir romain, il exerça pendant plus de trente ans une fonction qu'on pourrait appeler de méta-directeur de conscience. Pourtant, attaquant l'héliocentrisme, il soutient une théorie hétérodoxe des indivisibles ; voulant faire un sort à la grandeur montante de Descartes, il accorde aux cartésiens tout ce qu'ils demandaient sur les formes substantielles ; défendant Aristote, il condamne les scolastiques de son temps. Pour un auteur qui n'avait rien d'un libre-penseur, qui écrivait sous le soleil de l'orthodoxie et qui pratiquait professionnellement la censure, un certain nombre de choix théoriques étaient donc possibles : l'existence de fortes contraintes ne supprimait pas tout degré de liberté. Son œuvre devrait en ce sens nous garder d'un double écueil : croire que tout énoncé est possible à tout moment pourvu qu'il soit vrai ; s'imaginer que tout énoncé est déterminé par les contraintes des temps et des lieux - là encore, il faut tenir les deux bouts.

${ }^{71}$ Ordinatio pro studiis superioribus, op. cit., p. 90. 